\title{
Prevalence and clinical characteristics of remission during treatment in generalized anxiety
}

\author{
Marianne Destoop1, Filip van den Eede ${ }^{1,2}$, Marc Ansseau 3 , Adelin Albert ${ }^{4}$, Sophie \\ Vanbelle ${ }^{4}$, Annick Mignon ${ }^{5}$, Patricia Slachmuylders ${ }^{5}$ \& Bernard Sabbe ${ }^{1,6}$
}

${ }^{1}$ Collaborative Antwerp Psychiatric Research Institute (CAPRI), University of Antwerp, Universiteitsplein Wilrijk, Antwerp, Belgium,

${ }^{2}$ Department of Psychiatry, Antwerp University Hospital (UZA), Wilrijkstraat Edegem, Antwerp, Belgium,

${ }^{3}$ Department of Psychiatry, University of Liège (ULG), CHU du Sart Tilman (B35), Liège, Belgium,

${ }^{4}$ Department of Medical Informatics and Biostatistics, University of Liège (ULG), CHU Sart Tilman (B23),

Liège, Belgium,

${ }_{5}^{5}$ Pfizer SA/NV, Pleinlaan, Brussels, Belgium, and

${ }^{6}$ Psychiatric Hospital Sint-Norbertus, Stationsstraat Duffel, Belgium

KEYWORDS: Generalized anxiety, remission, prevalence, remission-related factors, remissioninhibiting factors

\begin{abstract}
Objective. Although the remission criteria for generalized anxiety are well defined, there is not much data available on the point prevalence of remission. The Measuring Impact of Remission in Anxiety Disorders in Belgium (MIRABEL) study is a naturalistic study designed to document the point prevalence of remission in patients treated for general anxiety and potential factors affecting this prevalence. Methods. The study population consisted of 618 adult outpatients being treated for generalized anxiety. The sample is defined by the key symptoms of generalized anxiety disorder rather than by fulfilling the exact DSM-IV- TR diagnostic criteria. Remission was defined as a Hamilton Anxiety Scale (HAM-A) score of less than or equal to 7. To reduce the interrater reliability, the HAM-A was assessed by the attending physicians who had no specific training. Factors investigated as possibly related to remission included sociodemographic, disease and treatment characteristics. Results. The point prevalence of remission in the study population was estimated at $13.3 \%$. Remission prevalence varied with occupational status and severity of the current anxiety episode. Remission prevalence was lower in the presence of comorbidity and was proportional to the number of comorbid symptoms. Remitters took fewer medications but were treated longer. Remission prevalence was higher in patients who were taking antidepressants, but was lower in patients who were taking sedatives. Conclusions. These findings underline the poor prognosis of generalized anxiety.
\end{abstract}




\section{Introduction}

Generalized anxiety is one of the most common anxiety disorders with a 12-month prevalence of 2.0 (Kessler et al. 2012) and a point prevalence of 13.4\% in Belgian primary care (Ansseau et al. 2008). The burden of generalized anxiety on society is substantial, not only due to the direct costs of treatment but also due to the indirect costs resulting from the impaired functioning in all aspects of life (Hoffman et al. 2008).

In the treatment of generalized anxiety, both cognitive psychotherapy and pharmacotherapy have been demonstrated to be effective (Davidson 2009; Bandelow et al. 2012). It is acknowledged that in generalized anxiety remission should be the primary treatment goal, and remission criteria have been established and implemented in clinical trials (Ballenger 2001; Doyle and Pollack 2003).

Despite the fact that generalized anxiety is a common and treatable disorder and the fact that remission criteria are well described, data do not seem to exist on the prevalence of remission in "real world" clinical settings. To amend this lack, the Measuring Impact of Remission in Anxiety disorders in Belgium (the MIRABEL) study was launched and the first results were reported in this paper. The study is primarily aimed at establishing remission rates in patients currently treated for generalized anxiety both in primary and psychiatric care settings. In addition, the purpose of this study was to analyse the potential impact of sociodemographic, disease and treatment factors on the point prevalence of remission.

\section{Methods and materials}

\section{OVERALL STUDY DESIGN AND PARTICIPANTS}

MIRABEL is an open-label, cross-sectional, observational, point-prevalence study of remission in adult patients currently receiving treatment for generalized anxiety. General practitioners (GPs) and psychiatrists in Belgium were invited to screen their patients with generalized anxiety during a consultation between October 2006 and December 2007. In order to be included in the study, patients had to satisfy the following criteria: (1) 18 years of age and over, (2) outpatient status, (3) treatment for generalized anxiety, and (4) written informed consent. Although not a mandatory criterion, we aimed to include patients who had been in treatment for a minimum of 3 and a maximum of 12 months, since the focus of the study was on patients in the acute phase of their disease. Treatments remained entirely at the discretion of the attending physicians. The research was conducted in accordance with the current version of the Declaration of Helsinki.

\section{ASSESSMENT PROCEDURE AND TOOLS}

As required by the protocol, having obtained written informed consent from their patient, all participating physicians used the same case report form (CRF) to collect and verify the patient information. To ensure that the data collected were accurate, consistent, complete, and reliable, a 
representative of the sponsor paid periodic visits to the investigation site while the study was in progress and reviewed the data thoroughly. All missing, contradictory, and critical data were identified and then were clarified by the investigator(s).

Symptom severity was assessed by the Hamilton Anxiety Scale (HAM-A) (Hamilton 1959), an observer-rated inventory consisting of 14 items, each gauging the severity of a specified set of symptoms on a 5-point Likert scale ranging from 0 (not present) to 4 (severe). As it was our goal to determine the prevalence of remission in patients with generalized anxiety receiving treatment, the HAM-A variable used in the definition of remission was taken as the primary variable. By definition, we categorized a patient as being in remission if HAM-A less than or equal to $7(7,9)$. No specific training was provided for the participating physicians. However, an investigator guide, including a short manual for the HAM-A, was provided.

To ease the investigator in verifying the symptoms of generalized anxiety and in recording the comorbid symptoms, the CRF captured the specific generalized anxiety symptoms (i.e., excessive anxiety and worry for at least 6 months, difficulty to control the worry, fatigue, difficulty concentrating, irritability, muscle tension, and insomnia) as well as the specific symptoms of depression (i.e. depressive mood and anhedonia) and other anxiety disorders (panic disorder, agoraphobia, specific phobia, social phobia, obsessive-compulsive disorder, or posttraumatic stress disorder).

Other variables that are likely to influence remission rates were also recorded for each patient; sociodemographic, disease and treatment factors, individual scores on the 14-item, and selfreported Hospital Anxiety and Depression (HADS) reflecting comorbidity with depression. Selfrating scales offer the possibility to find out whether the patients and physicians rate the symptoms in the same way. Since the exact duration of the previous generalized anxiety episodes was not assessed in the CRF, the number of previous episodes was not involved in the results.

\section{STATISTICAL ANALYSIS}

Quantitative variables and scores were expressed as mean \pm standard deviation (SD), whereas categorical variables were described in a frequency table. Prevalence estimates (\%) were associated with their 95\% confidence intervals (95\% CI). Mean values were compared by oneway analysis of variance (ANOVA) or Student's t-test, and proportions by the chi-squared test for contingency tables. The nonparametric Kruskal-Wallis and Wilcoxon tests were used if normality assumptions were not fulfilled. Cut-off points between remitters and nonremitters were obtained by logistic regression analysis. Calculations were always carried out on the maximum number of data available, and the missing data were not replaced. Results were considered to be significant at the 5\% critical level $(\mathrm{p}<0.05)$. Data analyses were carried out using SAS (version 9.1 for Windows) and S-PLUS (version 6.2) statistical packages.

Assuming a point prevalence of remission of generalized anxiety between $20 \%$ and $40 \%$ as reported in the literature (Kjernisted and Bleau 2004; Ballenger 2004), a sample size of 900 patients would ensure a statistical precision of less than or equal to $5 \%$ in the prevalence estimate with a $95 \%$ confidence. 


\section{Results}

\section{REMISSION RATE}

A total of 618 patients with generalized anxiety were included in the study and were recruited by 211 physicians (157 GPs and 54 psychiatrists). Among these patients, 365 (59.1\%) were treated by a GP (primary care) and 253 (40.9\%) by a psychiatrist (secondary care). The mean number of patients included by GPs was $5.0 \pm 1.1$ patients (range: $1-7$ ) while the mean number included by psychiatrists was $7.4 \pm 3.5$ patients (range: $1-13$ ) which differed significantly $(t(43.29)=-4.43$, $p$ $<0.001)$. HAM-A scores were available for 607 patients $(98.2 \%)$. Remission (HAM-A $\leq 7$ ) was observed in 81 subjects, yielding a point prevalence of 13.3\% (95\% CI: 10.6\%- 16.1\%). The HAMA mean score was $19.3 \pm 9.5$. Remission rates did not differ in primary and psychiatric care (12.8\% vs. $14.1 \%, \mathrm{p}=0.64)$.

\section{DEMOGRAPHY}

The demographic characteristics of the study population are displayed in

Table I. Except for occupational status $\left(p<0.0001, \chi^{2}(1)=31.98\right)$, characteristics of remitters and non-remitters were similar. Unfortunately demographic variables are missing in up to $1.3 \%$ of the patients. 
Table 1. Demographic characteristics of the patient population with generalized anxiety $(n=618)$ and comparison between remitters and non-remitters. Percentages are given in parentheses.

\begin{tabular}{|c|c|c|c|c|c|}
\hline \multirow[b]{2}{*}{ Variable } & \multirow[b]{2}{*}{ Total } & \multicolumn{2}{|c|}{$\begin{array}{c}\text { Remission } \\
\text { (HAM-A } \\
\leq 7)\end{array}$} & \multirow[b]{2}{*}{$\mathrm{p}$-value } & \multirow[b]{2}{*}{ Test statistic } \\
\hline & & No & Yes & & \\
\hline Age (yrs) Mean \pm SD & $46.8 \pm 14.3$ & $46.7 \pm 14.3$ & $47 \pm$ & 0.83 & $\mathrm{~T}=0.21$ \\
\hline \multicolumn{6}{|l|}{ Gender } \\
\hline $\begin{array}{l}\text { Female } \\
\text { Male }\end{array}$ & $\begin{array}{l}402(65.9) \\
208(34.1)\end{array}$ & $\begin{array}{l}341(86.5) \\
178(86.8)\end{array}$ & $\begin{array}{l}53 \\
27\end{array}$ & 0.92 & $\chi^{2}=0.0092$ \\
\hline \multicolumn{6}{|l|}{ Native language } \\
\hline $\begin{array}{l}\text { Dutch } \\
\text { French } \\
\text { Living status }\end{array}$ & $\begin{array}{l}291(47.1) \\
327(52.9)\end{array}$ & $\begin{array}{l}244(85.3) \\
282(87.9)\end{array}$ & $\begin{array}{l}42 \\
39 \\
(12.1)\end{array}$ & 0.36 & $\chi^{2}=0.84$ \\
\hline Single, independent & $152(24.8)$ & $130(87.2)$ & & 0.83 & $\chi^{2}=0.046$ \\
\hline With partner/family & $461(75.2)$ & $393(86.6)$ & $\hat{6} \hat{1}$ & & \\
\hline \multicolumn{6}{|l|}{ Education } \\
\hline No formal diploma & $14(2.3)$ & $13(92.9)$ & $1(7.1)$ & 0.70 & $\chi^{2}=2.98$ \\
\hline Primary & $52(8.5)$ & $46(88.5)$ & 6 & & \\
\hline Lower secondary & $202(33.0)$ & $173(88.3)$ & 23 & & \\
\hline Upper secondary & $143(23.4)$ & $122(86.5)$ & 19 & & \\
\hline Higher education & $154(25.2)$ & $129(84.9)$ & 23 & & \\
\hline $\begin{array}{l}\text { University Occupational } \\
\text { status }\end{array}$ & $47(7.7)$ & $37(80.4)$ & $\begin{array}{l}9 \\
(19.6)\end{array}$ & & \\
\hline No own income & $50(8.1)$ & $33(66)$ & & $<0.0001$ & $\chi^{2}=31.98$ \\
\hline Allowance (any) & $150(24.4)$ & $140(95.2)$ & $7(4.8)$ & & \\
\hline Self-employed & $38(6.2)$ & $34(89.5)$ & 4 & & \\
\hline Blue collar worker & $84(13.7)$ & $75(90.4)$ & $8(9.6)$ & & \\
\hline White collar worker & $145(23.6)$ & $119(83.8)$ & 23 & & \\
\hline Middle management & $30(4.9)$ & $26(89.7)$ & 3 & & \\
\hline Higher management & $12(2.0)$ & $9(75.0)$ & $3(25.0)$ & & \\
\hline Retired & $106(17.2)$ & $87(84.5)$ & $16(15.5)$ & & \\
\hline
\end{tabular}

\section{DISEASE FACTORS}

One hundred and sixty-seven (27.4\%) patients fulfilled the exact diagnostic criteria according the DSM-IV-TR for generalized anxiety disorder, while the majority of the other patients showed the symptoms as described in the DSM- IV-TR; the most prevalent symptoms were excessive anxiety and worry for at least 6 months (81.9\%), insomnia (73.1\%), difficulty to control the worry (69.0\%), fatigue $(67.0 \%)$, irritability (63.4\%), difficulty concentrating (61.8\%), restlessness (53.9\%) and muscle tension (47.3\%), respectively. Forty-five (10\%) patients presented all symptoms. The mean number of symptoms per patient was $5.2 \pm 1.8$.

As seen in Table II, the initial severity of the current generalized anxiety episode was markedly different in remitters and non-remitters $(p<0.0001)$. The mean number of symptoms was higher for patients treated by a psychiatrist compared to patients treated by a GP ( $6.4 \pm 1.3$ vs. $4.8 \pm 1.7, \mathrm{p}>0.0001)$. Patients with severe generalized anxiety disorder were more often seen by a psychiatrist than by a GP $(32.0 \%$ vs. $20.2 \%, \mathrm{p}=0.0015)$. Data on the initial severity of the current generalized anxiety episode were missing in $0.6 \%$. 
Globally, $88.2 \%$ of the patients presented with comorbid symptoms in the two weeks preceding the assessment. About $67.5 \%$ of the patients reported depressive mood and anhedonia and $53.1 \%$ of the patients had typical symptoms of other anxiety disorders (panic disorder, agoraphobia, specific phobia, social phobia, obsessive compulsive disorder, or posttraumatic stress disorder), while $34.1 \%$ (211) of the patients presented with both depressive mood/anhedonia and core symptoms of other anxiety disorders. The patients in remission had fewer comorbid symptoms than the non-remitters $(0.9 \pm 0.9$ vs. $2.0 \pm 1.3, \mathrm{p}<0.0001, \mathrm{~T}(1)=9.05)$. Accordingly, the prevalence of remission was low when comorbid symptoms were present $(43.1 \%$ vs. $9.4 \%, \mathrm{p}<0.0001, \mathrm{~T}(1)=62.36)$.

The mean HADS (assessing comorbidity with depression scale) anxiety score was $12.1 \pm 4.5$ (range: $0-21$ ) and the mean HADS depression score was $9.3 \pm 4.8$ (range: 0-21). Remitters showed less severe symptoms of anxiety $(6.6 \pm 4.0$ vs. $13.0 \pm 3.9, \mathrm{p}<0.0001, \mathrm{~T}(1)=13.59)$ and of depression ( $4.1 \pm 3.5$ vs. $10.1 \pm 4.5, \mathrm{p}<0.0001, \mathrm{~T}(1)=13.95)$ than that of non-remitters. The distributions of the HADS anxiety and depression scores for the two groups are displayed in Figure 1. The cut-off points for the HADS were found to be equal to 9.0 for anxiety and 5.9 for depression, respectively. In other words a HADS anxiety score higher than 9 was seen more frequently in nonremitters than in remitters and similarly for a HADS depression score higher than 5.9. Furthermore, the HADS anxiety and depression were correlated with the HAM-A score $(r=0.62, p<0.0001$ and $r$ $=0.58, \mathrm{p}<0.0001)$. The HADS anxiety and depression scores were missing in $1.0 \%$ and $0.6 \%$ of the participants, respectively.

Table 2. Disease factors in the patients population with generalized anxiety $(n=618)$ and comparison between remitters and non-remitters. Percentages are given in parentheses.

\begin{tabular}{|c|c|c|c|c|c|}
\hline \multicolumn{5}{|c|}{$\begin{array}{c}\text { Remission } \\
\text { (HAM- } \\
\text { A } \leq 7)\end{array}$} & \\
\hline Variable & Total & Yes & p-value & & \\
\hline \multicolumn{6}{|c|}{ Initial severity of the current GAD episode } \\
\hline $\begin{array}{l}\text { Mild } \\
\text { Moderate } \\
\text { Severe }\end{array}$ & $\begin{array}{r}75(12.4) \\
385(62.6) \\
154(25.0)\end{array}$ & $\begin{array}{r}53(69.7) \\
338(89.9) \\
132(86.8)\end{array}$ & $\begin{array}{l}23(30.3) \\
38(10.1) \\
20(13.2)\end{array}$ & $<0.0001$ & $\chi^{2}=22.13$ \\
\hline
\end{tabular}
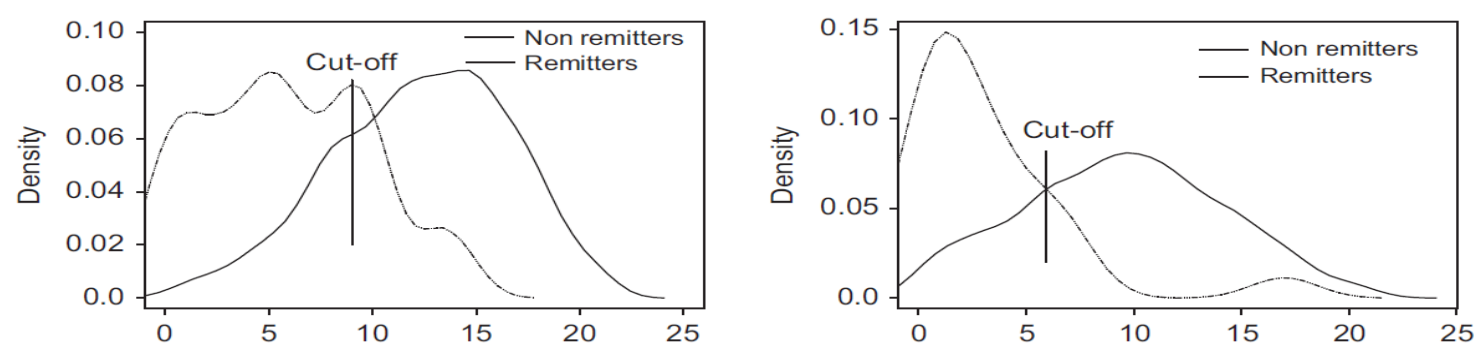

Figure 1. Distribution of the HADS anxiety score (left) and HADS depression score (right) in remitters and non-remitters. Cut-off points equal 9 for HADS anxiety and 5.9 for HADS depression. 


\section{TREATMENT FACTORS}

Treatment, consisting of medication, psychotherapy, or both, was initiated by GPs in $62.3 \%$ of the cases and by psychiatrists in $36.2 \%$. Medication was prescribed to $96.6 \%$ of the patients (593); with combinations of antidepressants and anxiolytics for $36.6 \%$, antidepressants alone for $25.7 \%$, and antidepressants, anxiolytics, and other medication for $7.9 \%$ being the most frequent. Other medications consisted of ant convulsants, antipsychotics, buspirone, and propanolol; and also passiflora, valerian and fentanyl. Overall 71.3\%(438) of the patients received psychotherapy (not further specified), delivered respectively by psychiatrists (45.0\%), GPs (36.8\%), and psychologists (24.4\%).

Table III gives an overview of the duration and type of treatment, as well as the number and type of prescribed drugs, globally and for remitters and non-remitters. Interestingly, in the remission group, the proportion of patients taking anxiolytics was lower than in the group of non-remitters ( $8.5 \%$ vs. $\left.20.1 \%, p<0.0001, \chi^{2}(1)=17.13\right)$, while the converse occurred for patients taking antidepressants $\left(14.7 \%\right.$ vs. $\left.4.0 \%, p=0.011, \chi^{2}(1)=6.46\right)$. The prevalence of remission did not differ between medicated and nonmedicated patients $\left(13.4 \%\right.$ vs.12.0\%, $\left.\mathrm{p}=0.84, \chi^{2}(1)=0.041\right)$, nor between patients receiving psychotherapy and those who did not $(13.6 \%$ vs. $13.0 \%, p=0.85$, $\left.\chi^{2}(1)=0.035\right)$. GPs more often initiated therapy with medication alone $(36.2 \%$ versus $17.9 \%)$, while psychiatrists more often initiated psychotherapy alone or a combination of medication and psychotherapy $(6.0 \%$ versus $1.7 \%$ and $76.2 \%$ versus $62.2 \%, \mathrm{p}<0.0001)$. GPs more often prescribed anxiolytics $(62.2 \%$ vs $52.2 \%, \mathrm{p}=0.013)$, while psychiatrists more often prescribed other medication $(26.5 \%$ vs. $10.7 \%, \mathrm{p}<0.0001)$. Treatment duration was shorter in primary-care settings ( 4.8 months \pm 4.7 vs. 6.1 months $\pm 4.9, \mathrm{p}=0.0016$ ), while the number of prescribed drugs did not differ between the two groups. Data about the treatment duration were missing in $4.5 \%$, while no data were missing about the type of treatment. 
Published in : International Journal of Psychiatry in Clinical Practice (2013), vol. 17, $n^{\circ} 2, p p$. 90-97

DOI:10.3109/13651501.2013.784789

Status : Postprint (Author's version)

Table 3. Treatment factors in the patients population $(n=618)$ and comparison between remitters and non-remitters. Percentages are given in parentheses.

\begin{tabular}{|c|c|c|c|c|c|}
\hline \multirow[b]{2}{*}{ Variable } & \multirow[b]{2}{*}{ Total } & \multicolumn{2}{|c|}{ Remission (HAM-A $\leq 7$ ) } & \multirow[b]{2}{*}{ p-value } & \multirow[b]{2}{*}{ Test statistic } \\
\hline & & No & Yes & & \\
\hline \multicolumn{6}{|c|}{ Treatment duration (months) } \\
\hline Mean \pm SD & $5.4 \pm 4.8$ & $5.3 \pm 5.0$ & $6.3 \pm 3.4$ & 0.019 & $\mathrm{~T}=2.37$ \\
\hline$\leq 3$ & $158(26.8)$ & $141(92.2)$ & $12(7.8)$ & 0.12 & $\chi^{2}=8.68$ \\
\hline $3-4$ & $105(17.8)$ & $90(87.4)$ & $13(12.6)$ & & \\
\hline $4-6$ & $132(22.4)$ & $109(83.8)$ & $21(16.2)$ & & \\
\hline $6-9$ & $92(15.6)$ & 77 (83.7) & $15(16.3)$ & & \\
\hline $9-12$ & $84(14.2)$ & $67(79.8)$ & $17(20.2)$ & & \\
\hline$>12$ & $19(3.2)$ & $16(84.2)$ & $3(15.8)$ & & \\
\hline \multicolumn{6}{|l|}{ Type of treatment } \\
\hline Medication & $176(28.7)$ & $147(87)$ & $22(13)$ & 0.98 & $\chi^{2}=0.98$ \\
\hline Psychotherapy & $21(3.4)$ & $18(85.7)$ & $3(14.3)$ & & \\
\hline Both & $417(67.9)$ & 357 (86.4) & $56(13.6)$ & & \\
\hline \multicolumn{6}{|c|}{ Number of prescription drugs } \\
\hline Mean \pm SD & $2.1 \pm 1.1$ & $2.1 \pm 1.1$ & $1.8 \pm 1$ & 0.0039 & $\mathrm{~T}=2.9$ \\
\hline \multicolumn{6}{|c|}{ Type of medication } \\
\hline \multicolumn{6}{|l|}{ Antidepressant } \\
\hline No & $77(12.5)$ & $72(96)$ & $3(4)$ & 0.011 & $\chi^{2}=6.46$ \\
\hline Yes & $541(87.5)$ & $454(85.3)$ & $78(14.7)$ & & \\
\hline \multicolumn{6}{|l|}{ Anxiolytic } \\
\hline No & $259(41.9)$ & $203(79.9)$ & $51(20.1)$ & $<0.0001$ & $\chi^{2}=17.13$ \\
\hline Yes & $359(58.1)$ & $323(91.5)$ & $30(8.5)$ & & \\
\hline \multicolumn{6}{|l|}{ Hypnotic } \\
\hline No & $541(87.5)$ & $456(85.7)$ & $76(14.3)$ & 0.069 & $\chi^{2}=3.30$ \\
\hline Yes & $77(12.5)$ & 70 (93.3) & $5(6.7)$ & & \\
\hline \multicolumn{6}{|l|}{ Other } \\
\hline No & $512(82.9)$ & $432(86.1)$ & 70 (13.9) & 0.34 & $\chi^{2}=0.9$ \\
\hline Yes & $106(17.2)$ & 94 (89.5) & $11(10.5)$ & & \\
\hline
\end{tabular}

\section{Discussion}

The present observational study aimed to establish the point prevalence of remission in patients with generalized anxiety during their first year of treatment delivered in both general and specialized-care settings, and to identify factors potentially impacting remission. Remission (HAM-A $\leq 7$ ) prevalence amounted $13.3 \%$, and was found to be influenced by the occupational status of the patient and the severity of the current generalized anxiety episode, while diminishing proportionally with comorbid symptoms. The remitters had been in treatment for a longer time period, with lower remission rates in patients taking sedatives, but higher in those taking antidepressants. 


\section{DEMOGRAPHIC FACTORS}

A recent review of pharmacotherapy for generalized anxiety concluded that remission was attainable in over $50 \%$ of the patients treated (Davidson 2009). However, in line with Allgulander (2010), several considerations need to be addressed concerning the clinical trials reviewed. First, the demographic characteristics of most clinical-trial populations differ markedly from those reported here. The clinical-trial participants mostly ranged between 30 and 65 years with a mean age of 40 years, thus excluding elderly patients (Dubois et al. 2010). As this study incorporated patients over 65 years, the mean age was higher ( 46.8 years). This may partly explain the lower remission rate obtained, as older age is associated with poorer treatment outcome (Simon et al. 2006).

According to some studies, gender also tends to affect the course of generalized anxiety in that women are generally associated with lack of recovery (Rodriguez et al. 2006). Yet, in the present study, no gender-related differences in remission rate were found. In their 8-year follow-up study, Yonkers et al. (2000) also failed to find any such differences in the clinical course of generalized anxiety.

Socioeconomic characteristics have been reported as significant risk factors with substantial consequences on generalized anxiety (Ansseau et al. 2008; Hunt et al. 2002; Pollack 2009). In line with Yonkers et al. (2000), living status and educational level were not found to be related to remission in this study. By contrast, for the first time, a positive association was demonstrated between remission and less-deprived socioeconomic status in the study patients, which is consistent with the literature on depression where higher income or full employment was also found to be associated with higher remission rates (Warden et al. 2009).

\section{DISEASE FACTORS}

Most patients who were evaluated in this study suffered from a moderate episode of generalized anxiety, that is, they were still capable of functioning to some (acceptable) extent despite manifesting clear anxiety symptoms impacting their functioning. As in the studies of Yonkers et al. (2003) and Penninx et al. (2011), we found higher remission prevalence in patients experiencing a mild episode. Moreover, the severity of illness and impairment were inversely associated with the likelihood of remission and recovery.

Clinical trials tend to exclude patients with a comorbid psychiatric condition even though one of the main predictors for the clinical course of generalized anxiety is comorbidity (Weisberg 2009). Our results support this fact in that more than $40 \%$ of the patients without comorbidity achieved remission as opposed to those with comorbid symptoms (less than 10\%). Furthermore, anxiety and depressive symptoms measured by the HADS were correlated with HAM-A scores. It should be noted, however, that the number of comorbidity-free patients in the present study was very small $(n=50)$. The majority of our patients $(88.2 \%)$ had comorbid symptoms, reflecting the clinical reality that $90 \%$ of all patients with generalized anxiety present with at least one comorbid lifetime psychiatric disorder (Pollack 2009). The strength of the current study is thus its naturalistic design, permitting to gain more insight into remission rates in clinical practice as compared to those derived from clinical trials and experimental settings. 


\section{TREATMENT FACTORS}

A remission rate of $13.3 \%$ is less than placebo response in most clinical trials so that one can even suggest that no active treatment has been provided on all in the current study. Indeed, according to Tonks (2003), low remission rates can in general also be attributed to the fact that the proportion of patients receiving the most appropriate or effective treatment is allegedly small. In the current study, treatment was left entirely to the discretion of the treating physicians and no inquiry was made about the rationale of the choice of treatment. Although the great majority of the patients had been prescribed antidepressants, no data were available about prescribed dose and treatment duration. Similarly, no data about the type and duration of psychotherapy were assembled, which makes it hard to evaluate the quality of treatment in the current study. Furthermore, low remission rates could not only be attributed to inferior prescription behaviour of the physician, but also to noncompliance of the patient. No data on the medication compliance in the pharmacotherapy group of the current study are available.

In scientific literature, cognitive-behavioural therapy (CBT) and applied relaxation have been demonstrated to be more effective than placebo NICE (2011), but no systematic research is available showing CBT or applied relaxation to be comparable to other pharmacotherapy, nor a combination of CBT or applied relaxation and medication to be more effective than either standalone treatment NICE (2011).

Recovery rates of CBT and applied relaxation varied between 33\% and 60\% (Davidson 2009). We found no significant effects favoring one of the mentioned treatment schemes but, admittedly, the number of remitted patients receiving either psychotherapy or pharmacotherapy alone was very small.

As the remission rate was found to be lower in patients taking anxiolytics and higher in those taking antidepressants, we referred to the recent reviews of therapies specific for generalized anxiety by Allgulander (2009), Davidson (2009), and Bandelow et al. (2012) who concluded that the efficacy of both psychological treatment and pharmacotherapy was comparable, but that efficacy differed among medication classes. The overall effect size of all medications for generalized anxiety disorder over that of placebo was 0.39 Allgulander (2009). According to the NICE guidelines, the strongest evidence for antidepressants, especially for SSRIs and SNRIs, makes them the drugs of choice in the treatment of generalized anxiety NICE (2011). In a recent metaanalysis, evidence for antidepressants was based on a total of 27 randomized controlled trials with effect size between 0.24 and 0.78 (compared with placebo). In this mixed treatment comparison by Baldwin et al., some estimates of the relative merits of differing compounds in achieving response and remission was reported.

Benzodiazepines act more quickly and are, consequently, used more frequently (1) to attain immediate anxiolytic relief in the initiation of other treatments, (2) in episodes of symptom exacerbation, and (3) in treatment-resistant patients without a history of addiction Allgulander (2009). Long-term benzodiazepine use requires consistent monitoring for dependence or intolerance. Based on these facts, we may assume that patients taking anxiolytics during the study period suffered a severe episode or were more resistant to treatment, thus explaining their reduced likelihood of remission. To date, the most plausible explanation for the lower remission rate in the "anxiolytic" group remains the lower effectiveness of these types of agents in the 
treatment of generalized anxiety. Unfortunately, owing to the low remission rate in both medication groups, we were unable to run detailed group analyses.

The prevalence of remission was higher when treatment duration was longer, but longer treatment is a relative concept in our study as we mostly included patients who had received between 3 and 12 months of therapy. The remission prevalence in the patients treated longer than 12 months was lower than would be expected. Possibly, patients treated in excess of a year suffer from a more refractory disease type with residual symptoms in which remission is likely to occur. The remission group used fewer pharmacological agents, a well-known bias in observational studies: patients with the severest problems receive the most therapy, but are less likely to remit. Nevertheless, in their follow-up studies neither Bruce et al. (2005) nor Rodriguez et al. (2006) found any of the treatment factors to be predictive. The research reported was part of a naturalistic longitudinal study in which treatment effects were typically washed out and in which adjustment for treatment effects was complex.

Although treatment in primary care is less appropriate than in psychiatric care with GPs prescribing more anxiolytics and initiating less-often psychotherapy during a shorter treatment duration, remission rates did not differ between primary and psychiatric care. A possible explanation is that patients treated by a psychiatrist suffer from a more-severe version of generalized anxiety demonstrated in the current study by a higher number of symptoms and a more-severe episode. In anxiety disorders, in general, Ormal et al. (1994) and de Graaf et al. (2010) already demonstrated underdiagnosis (and consequently undertreatment) by GPs result in poor remission rates for anxiety disorders in general.

\section{LIMITATIONS}

The evaluated patients with generalized anxiety were receiving treatment and therefore differed from the general population suffering from generalized anxiety with respect to disease severity, persistence, and comorbidity. Angst et al. (2009) postulated that treated patients suffer more distress and persistent anxiety and are more impaired at work by their anxiety. They are symptomatic for prolonged periods and suffer more frequently from concomitant type-2 bipolar disorder, social phobia, and cannabis dependence/abuse. Thus, although our findings cannot be generalized to the overall population suffering from generalized anxiety, our study sample is still representative of the clinical population, since we recruited patients from both primary and secondary care. Moreover, the patient characteristics are highly dependent on the general clinical policy adopted by Belgian GPs and psychiatrists; for instance, the prescription rate in Belgium is high and so is the use of benzodiazepines.

Initially the study was designed to enrol 900 patients with generalized anxiety to allow for a reliable point-prevalence estimate of remission. In the end only 618 patients were recruited.

An investigator guide including instructions for the HAM-A was provided to the participating physicians instead of a specific training. The lack of a specific training can have important consequences for the interrater reliability. On the other hand, the HAM-A has been shown good interrater reliability, even among inexperienced raters (Hamilton 1959; Bech et al. 1984)

The present study primarily sought to evaluate patients having received 3-12 months' treatment. We chose a minimum of 3 months as a time point where an optimal effect of the treatment can 
first be expected. CBT and applied relaxation therapy usually consists of 12-15 weekly sessions. For pharmacotherapy with SSRIs or SNRIs, a response can be expected after 6-10 weeks NICE (2011). The fact that $26.8 \%$ of the study population had received less than or equal to 3 months of therapy can partly explain the low remission rates obtained. Furthermore, $3.2 \%$ of the patients had been in treatment for over 12 months, that is, beyond the acute phase of treatment. Such patients are more likely to suffer from a refractory disorder and residual symptoms making remission less probable. Overall, generalized anxiety runs a longitudinal course, involving recurrent remissions and recurrences. Since follow-up studies which monitored patients for 5 years Warden et al. (2009) and 12 years Bruce et al. (2005) reported remission rates of $38 \%$ and $58 \%$, respectively, the choice of treatment period in this study may also account for the low remission rate. Accordingly, we believe that the results reported are largely representative of the population of adult outpatients with generalized anxiety in their first year of treatment.

The participating physicians made no use of systematic diagnostic procedures. Patients were included if they were initially diagnosed by their physicians as suffering from generalized anxiety and if they were put under treatment for this reason. The dimension of generalized anxiety is questioned by use of a standardized symptom checklist during ongoing treatment, that is, retrospectively. Comorbid symptoms were questioned likewise which adds to the heterogeneity of the sample. Although a structural diagnostic interview might be the preferred method, it is almost never used in primary care and not systematically by psychiatrists. Moreover, the use of a structural diagnostic interview is not applicable in a cross-sectional design like ours, where prevalence of remission is measured, as it would preclude patients in (partial) remission. It should be noted, however, that patients who had already terminated their treatment were not included, which could also partially explain the low remission rate.

\section{Conclusion}

The present observational, non-interventional, cross-sectional study is one of the first to present results about the point prevalence of remission of generalized anxiety. The remission rate for Belgian patients with generalized anxiety during the first year of treatment amounted to $13.3 \%$, much lower than would be expected. These findings once again underscore the need to improve the care for patients with generalized anxiety and are relevant for the prediction of prognosis in the first year of treatment. The two main limitations of the study are the lack of systematic diagnostic interviews and the use of a clinical sample of patients treated from 3 to 12 months. Therefore the results are only representative for a clinical population defined by key symptoms of generalized anxiety during the first year of the treatment. Large-scale studies are further needed to analyse the remitters in more detail, especially focusing on the effects of different medication classes and various types of psychotherapy. 


\section{Key Points}

- We measured the point prevalence of remission in patients treated for generalized anxiety and examined which factors affect this prevalence. The point prevalence was estimated at $13.3 \%$.

- The prevalence of remission was lower when the socioeconomic status was more deprived, comorbid symptoms were present, and the current generalized anxiety episode was more severe.

- Medication use was also linked with remission prevalence. Remitters took fewer medications but were treated longer. Remission prevalence was higher in patients taking antidepressants than in those who did not, but was lower in patients taking sedatives.

\section{Acknowledgements}

The authors would like to thank all the participants for their cooperation, and Anne Morsel for her linguistic advice.

\section{Statement of interest}

Annick Mignon and Patricia Slachmuylders were full-time employees of Wyeth pharmaceuticals at the time of the development and implementation of the study. There are no conflicts of interest to be reported for any of the other authors. Wyeth pharmaceuticals was only associated with the elaboration of the protocol and the collection of the data and was not involved in the analysis and interpretation of data.

The study was partly supported by a grant from Wyeth Belgium and by a Research Fund of the University of Antwerp. 


\section{References}

Allgulander C. 2009. Generalized anxiety disorder: between now and DSM-V. Psychiatr Clin North Am 32:611-628.

Allgulander C. 2010. Novel approaches to treatment of generalized anxiety disorder. Curr Opin Psychiatry 23:37-42.

Angst J, Gamma A, Baldwin DS, Ajdacic-Gross V, Rössler W. 2009. The generalized anxiety spectrum: prevalence, onset, course and outcome. Eur Arch Psychiatry Clin Neurosci 259:37-45.

Ansseau M, Fischler B, Dierick M, Albert A, Leyman S, Mignon A. 2008. Socioeconomic correlates of generalized anxiety disorder and major depression in primary care: the GADIS II study (Generalized Anxiety and Depression Impact Survey II). Depress Anxiety 25:506-513.

Baldwin D, Woods R, Taylor D. 2011. Efficacy of drug treatments for generalised anxiety disorder: systematic review and meta-analysis. BMJ 342:d1199.

Ballenger JC. 2001. Treatment of anxiety disorders to remission. J Clin Psychiatry 62:5-9.

Ballenger JC. 2004. Remission rates in patients with anxiety disorders treated with paroxetine. J Clin Psychiatry 65:1696-1707.

Bandelow B, Sher L, Bunevicius R, Hollander E, Kasper S, Zohar J, Möller HJ; WFSBP Task Force on Mental Disorders in Primary Care, WFSBP Task Force on Anxiety Disorders, OCD and PTSD. 2012. Guidelines for the pharmacological treatment of anxiety disorders, obsessive-compulsive disorder and posttraumatic stress disorder in primary care. Int J Psychiatry Clin Pract 16:77-84.

Bech P, Grosby H, Husum B, Rafaelsen L. 1984. Generalized anxiety or depression measured by the Hamilton Anxiety Scale and the Melancholia Scale in patients before and after cardiac surgery. Psychopathology 17:253-263.

Bruce SE, Yonkers KA, Otto MW, Eisen JL, Weisberg RB, Pagano M. 2005. Influence of psychiatric comorbidity on recovery and recurrence in generalized anxiety disorder, social phobia, and panic disorder: a 12-year prospective study. Am J Psychiatry 162:1179-1187. Davidson JRT. 2009. First-line Pharmacotherapy approaches for generalized anxiety disorder. J Clin Psychiatry 70:25-31.

de Graaf R, ten Have M, van Dorsselaer S. De psychische gezondheid van de Nederlandse bevolking. NEMESIS-2: Opzet en eerste resultaten. Utrecht: Trimbos-instituut, 2010.

Doyle AC, Pollack MH. 2003. Establishment of remission criteria for anxiety disorders. J Clin Psychiatry 64:40-45.

Dubois O, Salamon R, Germain C, Poirier MF, Vaugeois C, Banwarth B. 2010. Balneotherapy versus paroxetine in the treatment of generalized anxiety disorder. Complement Ther Med 18: 1-7.

Hamilton M. 1959. The assessment of anxiety states by rating. Br J Med Psychol 32:50-55.

Hoffman DL, Dukes EM, Wittchen HU. 2008. Human and economic burden of generalized anxiety disorder. Depress Anxiety 25:72-90. Hunt C, Issakidis C, Andrews G. 2002. DSM-IV generalized anxiety disorder in the Australian national Survey of Mental Health and well-being. Psychol Med 32:649-659.

Kessler RC, Petukhova M, Sampson NA, Zaslavsky AM, Wittchen HU. 2012. Twelve-month and lifetime prevalence and lifetime morbid risk of anxiety and mood disorders in the United States. Int J Methods Psychiatr Res 21:169-184. 
Kjernisted KD, Bleau P. 2004. Long-term goals in the management of acute and chronis anxiety disorders. Can J Psychiatry 49:51S-63S. NICE clinical guideline 113. Generalised anxiety disorder and panic disorder (with or without agoraphobia) in adults. Developed by the National Collaborating Centre for Mental Health and the National Collaborating Centre for Primary Care. January 2011.

Ormel J, Von Korff M, Üstün TB, Pini S, Korten A, Oldehinkel T. 1994. Common mental disorders and disabilities across cultures: results from WHO-collaborative study on psychopathologic conditions in primary care. JAMA 272:1741-1748.

Penninx BW, Nolen WA, Lamers F, Zitman FG, Smit JH, Spinhoven P, et al. 2011. Two-year course of depressive and anxiety disorders: results from the Netherlands Study of Depression and Anxiety (NESDA). J Affect Disord 133:76-85.

Pollack MH. 2009. Refractory generalized anxiety disorder. J Clin Psychiatry 70:32-38.

Rodriguez BF, Weisberg RB, Pagano ME, Bruce SE, Spencer MA, Culpepper L. 2006. Characteristics and predictors of full and partial recovery from generalized anxiety disorder in primary care patients. J Nerv Ment Dis 194:91-97.

Simon NM, Zalta AK, Worthington JJ, Hoge EA, Christian KM, Stevens JC. 2006. Preliminary support for gender differences in response to fluoxetine for generalized anxiety disorder. Depress Anxiety 23:373376.

Tonks A. 2003. Treating generalised anxiety disorder. BMJ 326: 700-702.

Warden D, Rush AJ, Lesser IM, Thase ME, Balasubramani GK, Shores-Wilson K. 2009. Income and attrition in the treatment of depression: a STAR D report. Depress Anxiety 26:622-633.

Weisberg RB. 2009. Overview of generalized anxiety disorder: epidemiology, presentation and course. J Clin Psychiatry70:4-9.

Yonkers KA, Bruce SE, Dyck IR, Keller MB. 2003. Chronicity, relapse, and illness-course of panic disorder, social phobia, and generalized anxiety disorder: findings in men and woman from 8 years follow-up. Depress Anxiety 17:173-179.

Yonkers KA, Dyck IR, Warshaw M, Keller MB. 2000. Factors predicting the clinical course of generalised anxiety disorder. Br J Psychiatry 176:544-549.

Zigmond AS, Snaith RP. 1983. The hospital anxiety and depression scale. Acta Psychiatr Scand 67:361370. 